\title{
Communication
}

\section{A Cognitive Behavioral Therapy-Informed Self-Management Program for Acute Respiratory Failure Survivors: A Feasibility Study}

\author{
Megan M. Hosey ${ }^{1,2,3, *}$, Stephen T. Wegener ${ }^{1}$, Caroline Hinkle ${ }^{1,4}$ and Dale M. Needham ${ }^{1,2,3}$ (D) \\ 1 Department of Physical Medicine and Rehabilitation, Johns Hopkins School of Medicine, \\ Baltimore, MD 21287, USA; swegener@jhmi.edu (S.T.W.); chinkle2@jhmi.edu (C.H.); \\ dale.needham@jhmi.edu (D.M.N.) \\ 2 Department of Medicine, Division of Pulmonary and Critical Care Medicine, Johns Hopkins School \\ of Medicine, Baltimore, MD 21287, USA \\ 3 Outcomes after Critical Illness and Surgery (OACIS) Research Group, Johns Hopkins University, \\ Baltimore, MD 21287, USA \\ 4 Department of Psychology, University of Maryland, Baltimore County, Baltimore, MD 21250, USA \\ * Correspondence: mhosey@jhu.edu
}

Citation: Hosey, M.M.; Wegener, S.T.; Hinkle, C.; Needham, D.M.

A Cognitive Behavioral

Therapy-Informed Self-Management Program for Acute Respiratory Failure Survivors: A Feasibility Study. J. Clin. Med. 2021, 10, 872. https:// doi.org/10.3390/jcm10040872

Academic Editor: Shigeaki Inoue

Received: 28 December 2020

Accepted: 16 February 2021

Published: 20 February 2021

Publisher's Note: MDPI stays neutral with regard to jurisdictional claims in published maps and institutional affiliations.

Copyright: (c) 2021 by the authors. Licensee MDPI, Basel, Switzerland. This article is an open access article distributed under the terms and conditions of the Creative Commons Attribution (CC BY) license (https:// creativecommons.org/licenses/by/ $4.0 /)$.

\begin{abstract}
Background: The number of people surviving critical illness is rising rapidly around the globe. Survivorship comes at a cost, with approximately half of patients with acute respiratory failure (ARF) experiencing clinically significant symptoms of anxiety, and $32-40 \%$ of survivors having substantial anxiety symptoms in the months or years after hospitalization. Methods: This feasibility study reports on 11 consecutive ARF patients receiving up to six sessions of a psychological intervention for self-management of anxiety. Results: All 11 patients accepted and received the psychological intervention. Four patients did not fully complete all 6 sessions due to death $(n=1$, 2 sessions completed), and early hospital discharge ( $n=3$, patients completed 2, 3 and 5 sessions). The median (IQR) score (range: 0-100; minimal clinically important difference: 13) for the Visual Analog Scale-Anxiety (VAS-A) pre-intervention was $70(57,75)$ points. During the intervention, all 11 patients had a decrease in VAS-A, with a median (IQR) decrease of $44(19,48)$ points. Conclusions: This self-management intervention appears acceptable and feasible to implement among ARF patients during and after an ICU stay.
\end{abstract}

Keywords: critical care; critical care outcomes; intensive care units; anxiety; cognitive behavior therapies

\section{Introduction}

The number of survivors of acute respiratory failure (ARF) requiring mechanical ventilation in an intensive care unit (ICU) continues to rise around the globe [1]. However, survival comes at a "cost," with survivors frequently experiencing long-lasting impairments in mental health, including depression, anxiety, and Post-Traumatic Stress Disorder (PTSD) $[2,3]$. To help address these impairments, there have been changes in clinical practice in the ICU. Evidence-based guidelines for ARF patients recommend minimizing sedation to reduce associated side effects (e.g., delirium, prolonged bedrest) [4,5]. Given decreased sedation, evaluating and addressing psychological distress for ARF patients in the ICU is presumably more feasible and important to consider. Notably, approximately $50 \%$ of ARF patients experience clinically significant levels of anxiety in the intensive care unit (ICU) [6-9], including feelings of terror, fear of death, loss of control, and eroded sense of self [7,10-12]. Moreover, clinically significant symptoms can be long-lasting after ARF, with $32-40 \%$ of survivors having clinically significant anxiety symptoms enduring for months or years $[3,13]$.

Preliminary studies suggest that early non-pharmacological interventions increase self-efficacy and may reduce longer-term mental health symptoms in ICU survivors [14]. 
First, a pre-post evaluation of a non-specific psychotherapeutic intervention in the ICU was associated with substantially lower anxiety and depression symptoms at 12-month follow-up. However, the psychological interventions used were not defined and cannot be readily replicated in other centers [15]. Second, two case studies suggest that cognitive behavioral therapy (CBT)-informed treatments conducted by a psychologist can expedite liberation from mechanical ventilation [16]. Third, a pilot randomized-controlled trial (RCT) of a CBT-informed protocol, administered by psychologists on a burn unit, reported that survivors $(n=50)$ had significantly reduced anxiety symptoms and post-traumatic stress disorder (PTSD) at 1 month follow-up [17]. Fourth, a national, cluster-randomized RCT conducted in the UK evaluated a 3 session, nurse-implemented, CBT-informed intervention delivered during an ICU stay [8]. This RCT did not demonstrate improved PTSD symptoms at 6-month follow-up; However, this study demonstrated reduced anxiety symptoms for those who received all 3 treatment sessions. Lessons from this RCT suggest: (1) intervention dose may be an important consideration in psychological interventions, and (2) the intervention should be defined, refined, and iterated for efficacy by psychologists and mental health professionals prior to eventual transition to other health professionals [18]. Collectively, these initial studies offer promising preliminary results; however, their feasibility, acceptability, and efficacy is under-studied in ARF patients and in the ICU and hospital setting.

CBT-informed self-management interventions are non-pharmacological treatments that have been demonstrated to reduce anxiety and improve functional outcomes in many medical populations [19-22], including individuals with pulmonary conditions [23-25]. These interventions place patients at the center of their recovery by providing them the knowledge and skills to manage symptoms [26]. Since such interventions are focused on managing specific problems and symptoms, they must be tailored to specific medical populations and settings $[18,26]$. Once developed and manualized, these interventions can be implemented by trained non-psychologists, such as nurses, physical therapists, and peer supports [27-30].

Given the need for additional study in this area, we adapted and implemented a CBT- informed self-management protocol for ARF patients. The purpose of this study was to evaluate the feasibility and acceptability of this protocol when used in routine psychology consultation and treatment in the Medical Intensive Care Unit at the Johns Hopkins Hospital, in Baltimore USA.

\section{Methods Section}

This feasibility study included a convenience sample of 11 consecutive patients in a medical intensive care unit who received consultation, assessment and treatment for anxiety using the protocol by a licensed clinical psychologist ( $\mathrm{MMH}$; [31]) who was clinically working two days per week during a 6-month period (November 2018-April 2019). To be eligible for the intervention, patients were experiencing ARF with mechanical ventilation and not delirious, as per the Confusion Assessment Method-ICU (CAM-ICU) screening tool and assessment by the psychologist. See Table 1 for full eligibility criteria. Feasibility was defined as $>70 \%$ of intervention sessions completed, and $<15 \%$ drop-out (defined as patient request to discontinue) among survivors by hospital discharge. Acceptability was evaluated via patient agreement to initiate with the intervention and continued participation in each subsequent intervention session.

Efficacious, CBT-informed self-management interventions in other medical populations consist of a total of 3 to 10 sessions with common treatment components to provide a patient "toolbox" of disease-related education and coping skills. Self-management in acute respiratory failure (SMARF) is a 6-session intervention which was adapted from existing protocols to improve anxiety in acute respiratory failure survivors [19,32,33]. Specific adaptation was based on previous research and prior clinical feedback from ARF patients, including focus on strategies for managing anxiety during mechanical ventilator liberation, problem solving to obtain education about medical treatment and prognosis, and coping 
skills related to rehabilitation engagement and uncertain hospital environments. Each session has a consistent structure, including anxiety measurement at the beginning of each session via VAS-A, education about the session topic, application of skills, and suggested "putting it into practice" activities, allowing patients to practice skills between sessions. The SMARF protocol is shown in Table 2.

Table 1. Eligibility Criteria for Patients.

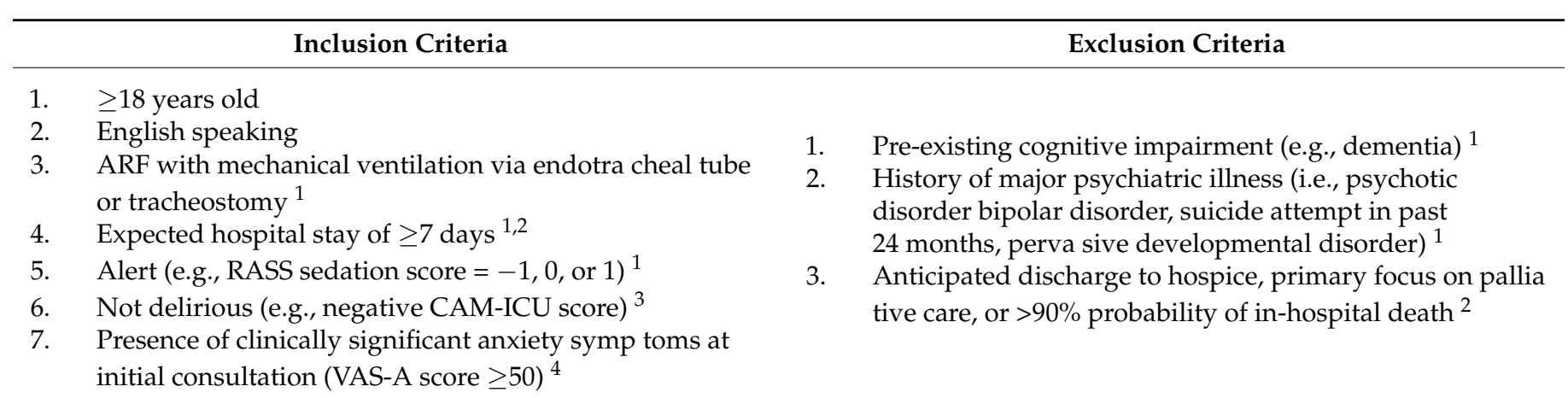

${ }^{1}$ At the time of initial psychology consultation. ${ }^{2}$ Determined by Medical Intensive Care Unit staff. ${ }^{3}$ Confusion Assessment MethodIntensive Care Unit. ${ }^{4}$ The Visual Analog Scale-Anxiety (VAS-A) ranges from "not anxious at all" (score $\left.=0\right)$ to "most anxious I have ever felt" $($ score $=100)$. Note: ARF = Acute Respiratory Failure; RASS = Richmond Agitation-Sedation Scale .

Table 2. Self-Management for Acute Respiratory Failure (SMARF) Intervention.

SMARF Session

1. Education \& Goal Setting

2. Elicit Concerns \& Problem Solving

3. Thought Restructuring

4. Relaxation training

5. Exposure

6. Transition out of Hospital

\section{Session Description}

Learning the relationship between medical events and changes in anxiety level; education about structure and format of sessions; set goals for SMARF treatment

Eliciting concerns about medical care (focusing on concerns within patient control); develop problem-solving skills \& established approaches, including SMART (Specific, Measurable, Attainable, Relevant, Time-sensitive) goals

Identifying the relationship between thoughts and anxiety, identifying \& challenging maladaptive thoughts and beliefs

Identifying the relation between anxiety and physiologic response, and acquiring relaxation skills (e.g., guided imagery, body scan, and breathing retraining)

Understanding fear-avoidance patterns; providing in-vivo exposure to anxiety provoking events

Providing education about anxiety and discharge from hospital; identifying "red flags" about levels of anxiety; discussing how to find help in the next settings

Patients had initial sessions in the medical intensive care unit (MICU) and were followed in the acute care hospital unit until the 6 sessions were completed. Delirium was assessed at each session and sessions were rescheduled if the patient was delirious. Sessions with intubated patients were conducted using individually tailored communication procedures, including talking tracheostomy devices, handwriting, communication boards, offering multiple choice and yes/no questions, and lip reading, depending on the needs, abilities and preferences of each patient. The interventionist participated in the SPEACS2 Communication Training Program [34]. Each session lasted approximate 30-40 min. This feasibility study was approved by the Johns Hopkins Institutional Review Board.

\section{Results}

The 11 patients were 55\% female and 55\% White (see Table 3). All 11 (100\%) patients accepted to initiate the SMARF intervention, with a goal to deliver the 6 SMARF sessions. Four patients did not complete all 6 sessions due to death ( $n=1,2$ sessions completed), and hospital discharge ( $n=3$, each patient completed 2, 3 and 5 sessions). All 11 ARF 
patients experienced delirium prior to the psychology consultation. One patient had a clinical deterioration and recurrence of delirium, forcing cancellation of all subsequent sessions, with eventual patient death. No other patients had episodes of delirium requiring cancellation or rescheduling of sessions. The VAS anxiety score (range: 0 to 100) was high immediately prior to the start of intervention (i.e., "baseline"), with a median (interquartile range [IQR]) score of $70(57,75)$. At the last SMARF session, all 11 patients experienced a decrease from baseline. In all patients, and in the subset of 7 patients who completed all 6 sessions, the median (IQR) decrease in VAS-A from baseline was $44(21,48)$ and 51 $(44,62)$ points, respectively (Table 4; Figure 1). No patient declined any of 54 SMARF intervention sessions.

Table 3. Characteristics of 11 Patients Receiving Self-Management Intervention.

\begin{tabular}{lc}
\hline \multicolumn{1}{c}{ Patient Characteristic } & Statistic \\
\hline Age, Median (IQR) Years & $48(32,69)$ \\
Female, N (\%) & $6(55)$ \\
Race/Ethnicity, N (\%) & $6(55)$ \\
$\quad$ White & $4(36)$ \\
$\quad$ Black & $1(9)$ \\
$\quad$ Hispanic Ethnicity & \\
ICU Admission Diagnosis Category, N (\%) & $8(73)$ \\
$\quad$ Acute Hypoxemia Respiratory Failure & $1(9)$ \\
$\quad$ Chronic Obstructive Pulmonary Disease & $1(9)$ \\
$\quad$ Sepsis & $1(9)$ \\
$\quad$ Gastrointestinal Bleed & $47(24,58)$ \\
ICU LOS, median (IQR) days & $51(30,121)$ \\
Hospital LOS, median (IQR) days & $19(13,21)$ \\
ICU LOS prior to start of intervention, median & \\
(IQR) days & $25(14,49)$ \\
Hospital LOS prior to start of intervention, & \\
median (IQR) days & \\
\hline Total days in ICU during hospital stay (i.e., includes any ICU re-admission). Note: IQR = Interquartile range; \\
ICU = Intensive Care Unit; LOS = Length of stay.
\end{tabular}

Table 4. Self-Management Intervention Data.

\begin{tabular}{lc}
\hline \multicolumn{1}{c}{ Intervention Result } & Statistic \\
\hline Agree to participate in intervention, N (\%) & $11(100)$ \\
Number of sessions completed per patient, median (IQR) & $6(3,6)$ \\
Pre-intervention VAS-A ${ }^{1}$ score, median (IQR) & $70(57,75)$ \\
Intervention sessions completed in ICU, N (\%) & $42(84 \%)$ \\
VAS-A $^{1}$ decrease from pre-intervention to final session for all patients, median (IQR) & $44(21,48)$ \\
VAS-A $^{1}$ decrease from pre-intervention to final session for patients receiving all 6 sessions, median (IQR) & $51(44,62)$ \\
\hline
\end{tabular}

${ }^{1}$ The Visual Analog Scale-Anxiety (VAS-A) ranges from "not anxious at all" (score = 0) to "most anxious I have ever felt" $($ score = 100). Note: IQR-Interquartile range. 


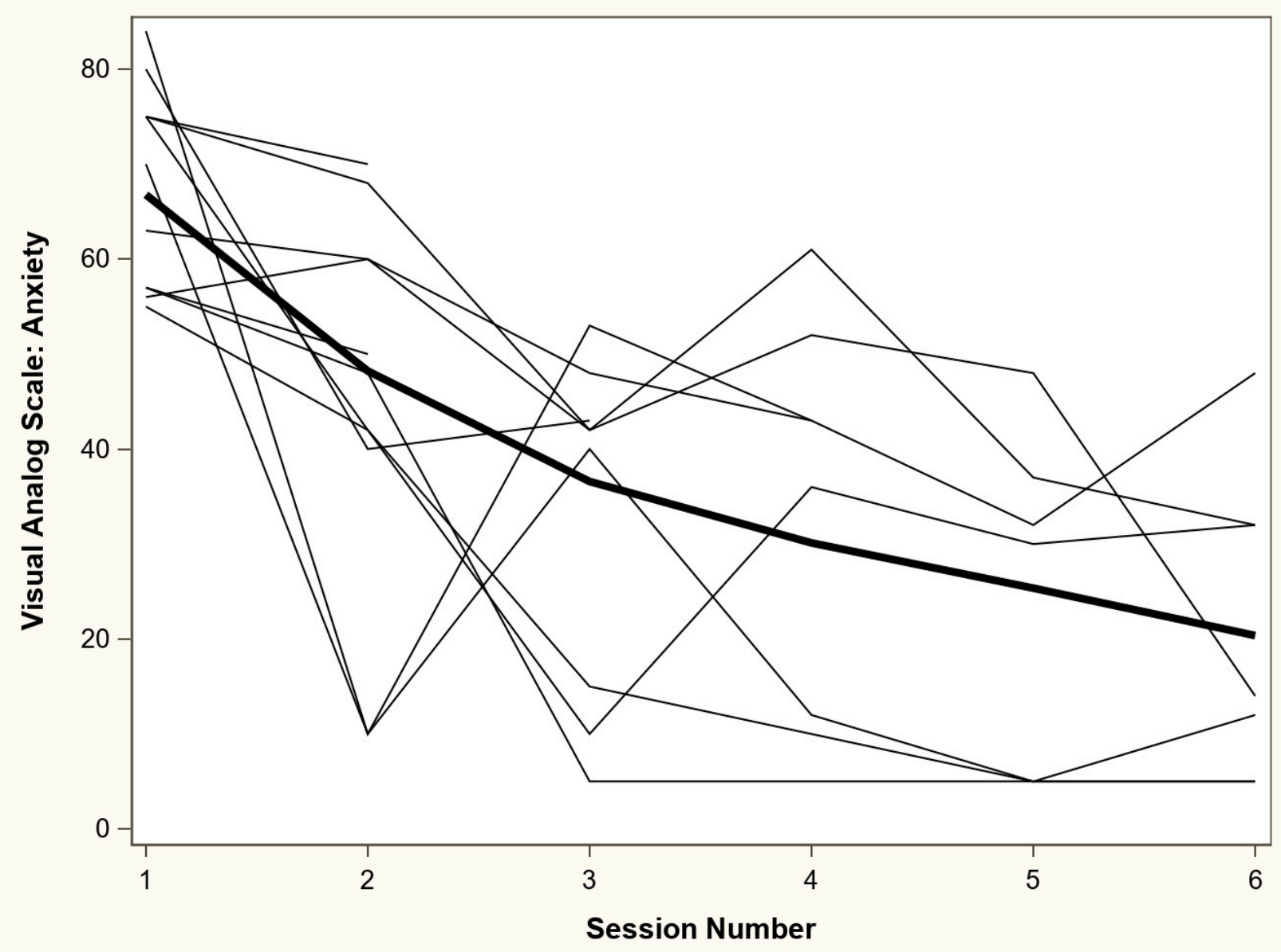

Figure 1. Visual Analog Scale-Anxiety (VAS-A) scores across self-management intervention session. VAS-A scores range from "not anxious at all" (score $=0)$ to "most anxious I have ever felt" (score $=100)$. The bold line represents a locally estimated scatterplot smoothing (LOESS) regression line across all data points, and all other lines represent values from each patient across each of the self-management sessions.

\section{Discussion}

This study evaluates the feasibility of a psychologist-delivered, CBT-informed, selfmanagement intervention for patients with ARF, delivered in the ICU and acute care unit as part of routine clinical care [31]. The 11 patients in this feasibility study had similar ratings of anxiety when compared to other cohorts of patients receiving non-pharmacological interventions for anxiety in the ICU [7-9]. Feasibility goals of $>70 \%$ of intervention sessions completed (across all patients), and $<15 \%$ drop-out of survivors (as defined by patient request to discontinue) by hospital discharge were met. No sessions were declined by patients, suggesting that they found the intervention acceptable. Based on this study, the SMARF intervention is feasible and acceptable to non-delirious patients without history of severe psychiatric or cognitive comorbidities. SMARF also demonstrates potential for improvement in anxiety symptoms. To evaluate the efficacy of the intervention, a randomized controlled trial is needed.

Patients in this case series had long ICU and hospital lengths of stay [8,35]. A longer length of stay may make psychological symptoms more apparent and provide time for consultation. In addition, patients with increased length of stay may be more likely to have problems that trigger psychology consultation [36].

Important considerations regarding feasibility of the intervention include delirium, which is common in ARF patients in the ICU [37]. Inattention, a cardinal feature of delirium, would prevent the necessary learning for patients to benefit from the intervention. Hence, 
patients only received the intervention once delirium resolved. Despite all patients having delirium prior to start of the intervention, only 1 of the 11 patients experienced delirium after start of the intervention that was associated with clinical deterioration that required cessation of the intervention and subsequent death. Another consideration regarding feasibility was availability of adequate time to conduct the 30-40 min intervention given that critically ill and hospitalized patients often have unpredictable schedules whereby medical interventions (e.g., medications administration, imaging, procedures) may interrupt SMARF sessions. Finally, fatigue is common in critically ill patients hospitalized patients. To help ensure feasibility of the intervention, the following strategies were helpful: (1) communicating regularly with the ICU and ward clinical staff about patient availability/scheduling and maintaining flexibility in scheduling sessions with an expectation that patients may require more than 1 attempt to initiate a session, (2) eliciting the patient's communication preferences (e.g., writing, lip reading) and (3) providing written materials to assist with recall and practice of skills learned during the session.

In this small case series, anxiety reduction was substantial and exceeded the minimum clinically important difference for the VAS-A instrument [38]. This preliminary observation may be important, as there is great need for effective non-pharmacological strategies for managing anxiety in the ICU and hospital. Despite guidelines suggesting reduced use of benzodiazepines and other sedating medications, hospital and ICU providers have not been provided with evidence or support for helpful alternatives [4]. Medications remain the most common means of reducing anxiety [6]. This can have important harmful effects including increased delirium, worsened anxiety, and lengthened ICU and hospital stays $[39,40]$.

This study has several important limitations. First, the small convenience sample, lack of a control group, and limited information about previous history of mental health symptoms and treatment preclude any definitive statements about the efficacy of the SMARF intervention. A second limitation is that most ICUs do not have access to a psychologist with expertise in care of ARF patients. However, experts (e.g., trained psychologists) must develop successful behavioral intervention protocols. Training and implementation by other healthcare providers or via technology is an important subsequent step after the initial development of an efficacious protocol [18]. Third, it is possible that the natural history of anxiety is a decline over time and that the intervention was not causally related to the observed reduction in VAS-A scores. In the future, it will be beneficial to obtain patient/stakeholder feedback about the appropriateness, timing, and duration of sessions.

\section{Conclusions}

The goal of this small study is to explore the acceptability and feasibility of a psychological intervention implemented in the ICU and acute care hospital unit to lay the foundation for future work evaluating the efficacy, effectiveness, and implementation of an intervention to improve long-term mental health outcomes for ARF survivors. The SMARF intervention, delivered in the ICU and acute care unit, is feasible and acceptable for non-delirious ARF patients. SMARF has the potential to improve self-management of anxiety in hospitalized ARF patients. Additional studies are needed to further evaluate and extend these findings.

Author Contributions: Conceptualization, M.M.H., S.T.W., D.M.N.; methodology, M.M.H., D.M.N.; formal analysis M.M.H.; writing-original draft preparation M.M.H., C.H., S.T.W., D.M.N. All authors have read and agreed to the published version of the manuscript.

Funding: This research was funded by the Parker B. Francis Foundation, grant number 134246.

Institutional Review Board Statement: The research was approved by the Johns Hopkins University School of Medicine Institutional Review Board (protocol number: NA_00048180, approved 8 April 2015).

Informed Consent Statement: Informed consent was waived for this study as data were collected from the medical record in the context of routine clinical care. 
Acknowledgments: The authors would like to acknowledge Earl Mantheiy, BA for his assistance data management and Lisa Aronson Friedman, Sc.M. for assistance in creating the figure.

Conflicts of Interest: The authors declare no conflict of interest. The funders had no role in the design of the study; in the collection, analyses, or interpretation of data; in the writing of the manuscript, or in the decision to publish the results.

\section{References}

1. Lilly, C.M.; Swami, S.; Liu, X.; Riker, R.R.; Badawi, O. Five-Year Trends of Critical Care Practice and Outcomes. Chest 2017, 152, 723-735. [CrossRef] [PubMed]

2. Needham, D.M.; Davidson, J.; Cohen, H.; Hopkins, R.O.; Weinert, C.; Wunsch, H.; Zawistowski, C.; Bemis-Dougherty, A.; Berney, S.C.; Bienvenu, O.J.; et al. Improving long-term outcomes after discharge from intensive care unit: Report from a stakeholders' conference. Crit. Care Med. 2012, 40, 502-509. [CrossRef]

3. Bienvenu, O.J.; Friedman, L.A.; Colantuoni, E.; Dinglas, V.D.; Sepulveda, K.A.; Mendez-Tellez, P.; Shanholz, C.; Pronovost, P.J.; Needham, D.M. Psychiatric symptoms after acute respiratory distress syndrome: A 5-year longitudinal study. Intensive Care Med. 2018, 44, 38-47. [CrossRef]

4. Devlin, J.W.; Skrobik, Y.; Gélinas, C.; Needham, D.M.; Slooter, A.J.C.; Pandharipande, P.P.; Watson, P.L.; Weinhouse, G.L.; Nunnally, M.E.; Rochwerg, B.; et al. Clinical Practice Guidelines for the Prevention and Management of Pain, Agitation/Sedation, Delirium, Immobility, and Sleep Disruption in Adult Patients in the ICU. Crit. Care Med. 2018, 46, e825-e873. [CrossRef] [PubMed]

5. Ely, E.W. The ABCDEF Bundle: Science and Philosophy of How ICU Liberation Serves Patients and Families. Crit. Care Med. 2017, 45, 321-330. [CrossRef]

6. May, A.D.; Parker, A.M.; Caldwell, E.S.; Hough, C.L.; Jutte, J.E.; Gonzalez, M.S.; Needham, D.M.; Hosey, M.M. ProviderDocumented Anxiety in the ICU: Prevalence, Risk Factors, and Associated Patient Outcomes. J. Intensive Care Med. 2020, 0885066620956564. [CrossRef]

7. Chlan, L.; Savik, K. Patterns of anxiety in critically ill patients receiving mechanical ventilatory support. Nurs. Res. 2011, 60, S50. [CrossRef] [PubMed]

8. Wade, D.M.; Mouncey, P.R.; Richards-Belle, A.; Wulff, J.; Harrison, D.A.; Sadique, M.Z.; Grieve, R.D.; Emerson, L.M.; Mason, A.J.; Aaronovitch, D.; et al. Effect of a Nurse-Led Preventive Psychological Intervention on Symptoms of Posttraumatic Stress Disorder Among Critically Ill Patients: A Randomized Clinical Trial. JAMA 2019, 321, 665-675. [CrossRef]

9. Karnatovskaia, L.V.; Schulte, P.J.; Philbrick, K.L.; Johnson, M.M.; Anderson, B.K.; Gajic, O.; Clark, M.M. Psychocognitive sequelae of critical illness and correlation with 3 months follow up. J. Crit. Care 2019, 52, 166-171. [CrossRef]

10. Wade, D.M.; Brewin, C.R.; Howell, D.C.J.; White, E.; Mythen, M.G.; Weinman, J.A. Intrusive memories of hallucinations and delusions in traumatized intensive care patients: An interview study. Br. J. Health Psychol. 2015, 20, 613-631. [CrossRef]

11. Agard, A.S.; Egerod, I.; Tonnesen, E.; Lomborg, K. Struggling for independence: A grounded theory study on convalescence of ICU survivors 12 months post ICU discharge. Intensive Crit Care Nurs. 2012, 28, 105-113. [CrossRef]

12. Hashem, M.D.; Nallagangula, A.; Nalamalapu, S.; Nunna, K.; Nausran, U.; Robinson, K.A.; Dinglas, V.D.; Needham, D.M.; Eakin, M.N. Patient outcomes after critical illness: A systematic review of qualitative studies following hospital discharge. Crit. Care 2016, 20. [CrossRef]

13. Nikayin, S.; Rabiee, A.; Hashem, M.D.; Huang, M.; Bienvenu, O.J.; Turnbull, A.E.; Needham, D.M. Anxiety symptoms in survivors of critical illness: A systematic review and meta-analysis. Gen. Hosp. Psychiatry 2016, 43, 23-29. [CrossRef]

14. Roberts, M.B.; Glaspey, L.J.; Mazzarelli, A.; Jones, C.W.; Kilgannon, H.J.; Trzeciak, S.; Roberts, B.W. Early Interventions for the Prevention of Posttraumatic Stress Symptoms in Survivors of Critical Illness: A Qualitative Systematic Review. Crit. Care Med. 2018, 46, 1328. [CrossRef] [PubMed]

15. Peris, A.; Bonizzoli, M.; Iozzelli, D.; Migliaccio, M.L.; Zagli, G.; Bacchereti, A.; Debolini, M.; Vannini, E.; Solaro, M.; Balzi, I.; et al. Early intra-intensive care unit psychological intervention promotes recovery from post traumatic stress disorders, anxiety and depression symptoms in critically ill patients. Crit. Care 2011, 15, R41. [CrossRef] [PubMed]

16. Cohen, J.; Gopal, A.; Roberts, K.J.; Anderson, E.; Siegel, A.M. Ventilator Dependent Patients Successfully Weaned with Cognitive Behavioral Therapy: A Case Series. Psychosomatics 2019. In Press. [CrossRef] [PubMed]

17. Fauerbach, J.A.; Gehrke, A.K.; Mason, S.T.; Gould, N.F.; Milner, S.M.; Caffrey, J. Cognitive Behavioral Therapy for Acute Posttrauma Distress: A Randomized, Controlled, Proof-of-Concept Study among Hospitalized Adults with Burns. Arch. Phys. Med. Rehabil. 2019. [CrossRef]

18. Czajkowski, S.M.; Powell, L.H.; Adler, N.; Naar-King, S.; Reynolds, K.D.; Hunter, C.M.; Laraia, B.; Olster, D.H.; Perna, F.M.; Peterson, J.C.; et al. From ideas to efficacy: The ORBIT model for developing behavioral treatments for chronic diseases. Health Psychol. Off. J. Div. Health Psychol. Am. Psychol. Assoc. 2015, 34, 971-982. [CrossRef]

19. Bradford, A.N.; Castillo, R.C.; Carlini, A.R.; Wegener, S.T.; Teter, H.J.; Mackenzie, E.J. The Trauma Survivors Network: Survive. Connect. Rebuild. J. Trauma Acute Care Surg. 2011, 70, 1557-1560. [CrossRef] [PubMed]

20. Wegener, S.T.; Mackenzie, E.J.; Ephraim, P.; Ehde, D.; Williams, R. Self-Management Improves Outcomes in Persons With Limb Loss. Arch. Phys. Med. Rehabil. 2009, 90, 373-380. [CrossRef] [PubMed] 
21. Lorig, K.R.; Sobel, D.S.; Stewart, A.L.; Brown, B.W.J.; Bandura, A.; Ritter, P.; Gonzalez, V.M.; Laurent, D.D.; Holman, H.R. Evidence Suggesting That a Chronic Disease Self-Management Program Can Improve Health Status While Reducing Hospitalization: A Randomized Trial. Med. Care 1999, 37, 5-14. [CrossRef] [PubMed]

22. Cadel, L.; DeLuca, C.; Hitzig, S.L.; Packer, T.L.; Lofters, A.K.; Patel, T.; Guilcher, S.J.T. Self-management of pain and depression in adults with spinal cord injury: A scoping review. J. Spinal Cord Med. 2018, 1-18. [CrossRef]

23. Lamers, F.; Jonkers, C.C.M.; Bosma, H.; Chavannes, N.H.; Knottnerus, J.A.; van Eijk, J.T. Improving Quality of Life in Depressed COPD Patients: Effectiveness of a Minimal Psychological Intervention. COPD J. Chronic Obstr. Pulm. Dis. 2010, 7, $315-322$. [CrossRef] [PubMed]

24. Heslop-Marshall, K.; Baker, C.; Carrick-Sen, D.; Newton, J.; Echevarria, C.; Stenton, C.; Jambon, M.; Gray, J.; Pearce, K.; Burns, G.; et al. Randomised controlled trial of cognitive behavioural therapy in COPD. ERJ Open Res. 2018, 4. [CrossRef]

25. Howard, C.; Dupont, S. 'The COPD breathlessness manual': A randomised controlled trial to test a cognitive-behavioural manual versus information booklets on health service use, mood and health status, in patients with chronic obstructive pulmonary disease. NPJ Prim. Care Respir. Med. 2014, 24, 1-8. [CrossRef]

26. Lorig, K.R.; Holman, H.R. Self-management education: History, definition, outcomes, and mechanisms. Ann. Behav. Med. 2003, 26, 7. [CrossRef]

27. Archer, K.R.; Devin, C.J.; Vanston, S.W.; Koyama, T.; Phillips, S.E.; George, S.Z.; McGirt, M.J.; Spengler, D.M.; Aaronson, O.S.; Cheng, J.S.; et al. Cognitive-Behavioral-Based Physical Therapy for Patients With Chronic Pain Undergoing Lumbar Spine Surgery: A Randomized Controlled Trial. J. Pain 2016, 17, 76-89. [CrossRef]

28. Lorig, K.R.; Hurwicz, M.-L.; Sobel, D.; Hobbs, M.; Ritter, P.L. A national dissemination of an evidence-based self-management program: A process evaluation study. Patient Educ. Couns. 2005, 59, 69-79. [CrossRef]

29. Druss, B.G.; Zhao, L.; von Esenwein, S.A.; Bona, J.R.; Fricks, L.; Jenkins-Tucker, S.; Sterling, E.; DiClemente, R.; Lorig, K. The Health and Recovery Peer (HARP) Program: A peer-led intervention to improve medical self-management for persons with serious mental illness. Schizophr. Res. 2010, 118, 264-270. [CrossRef]

30. Bodenheimer, T.; Lorig, K.; Holman, H.; Grumbach, K. Patient Self-management of Chronic Disease in Primary Care. JAMA 2002, 288, 2469-2475. [CrossRef] [PubMed]

31. Hosey, M.M.; Ali, M.K.; Mantheiy, E.C.; Albert, K.; Wegener, S.T.; Needham, D.M. Psychology consultation patterns in a medical intensive care unit: A brief report. Rehabil. Psychol. 2019, 64, 360-365. [CrossRef]

32. Spathis, A.; Booth, S.; Moffat, C.; Hurst, R.; Ryan, R.; Chin, C.; Burkin, J. The Breathing, Thinking, Functioning clinical model: A proposal to facilitate evidence-based breathlessness management in chronic respiratory disease. NPJ Prim. Care Respir. Med. 2017, 27, 1-6. [CrossRef] [PubMed]

33. Sperry, L. Treatment of Chronic Medical Conditions: Cognitive-Behavioral Therapy Strategies and Integrative Treatment Protocols; American Psychological Association: Washington, DC, USA, 2009; ISBN 978-1-4338-0389-5.

34. Happ, M.B.; Baumann, B.M.; Sawicki, J.; Tate, J.A.; George, E.L.; Barnato, A.E. SPEACS-2: Intensive Care Unit “Communication Rounds" with Speech Language Pathology. Geriatr. Nur. (Lond.) 2010, 31, 170-177. [CrossRef]

35. Chlan, L.L.; Weinert, C.R.; Heiderscheit, A.; Tracy, M.F.; Skaar, D.J.; Guttormson, J.L.; Savik, K. Effects of patient-directed music intervention on anxiety and sedative exposure in critically ill patients receiving mechanical ventilatory support: A randomized clinical trial. JAMA 2013, 309, 2335-2344. [CrossRef]

36. Bashar, F.R.; Vahedian-Azimi, A.; Hajiesmaeili, M.; Salesi, M.; Farzanegan, B.; Shojaei, S.; Goharani, R.; Madani, S.J.; Moghaddam, K.G.; Hatamian, S.; et al. Post-ICU psychological morbidity in very long ICU stay patients with ARDS and delirium. J. Crit. Care 2018, 43, 88-94. [CrossRef] [PubMed]

37. Girard, T.D.; Thompson, J.L.; Pandharipande, P.P.; Brummel, N.E.; Jackson, J.C.; Patel, M.B.; Hughes, C.G.; Chandrasekhar, R.; Pun, B.T.; Boehm, L.M.; et al. Clinical phenotypes of delirium during critical illness and severity of subsequent long-term cognitive impairment: A prospective cohort study. Lancet Respir. Med. 2018, 6, 213-222. [CrossRef]

38. Williams, V.S.; Morlock, R.J.; Feltner, D. Psychhometric evaluation of a visual analog scale for the assessment of anxiety. Health Qual. Life Outcomes 2010, 8, 8. [CrossRef]

39. Kok, L.; Slooter, A.J.; Hillegers, M.H.; van Dijk, D.; Veldhuijzen, D.S. Benzodiazepine Use and Neuropsychiatric Outcomes in the ICU: A Systematic Review*. Crit. Care Med. 2018, 46, 1673-1680. [CrossRef]

40. Pisani, M.A.; Murphy, T.E.; Araujo, K.L.B.; Slattum, P.; Van Ness, P.H.; Inouye, S.K. Benzodiazepine and opioid use and the duration of ICU delirium in an older population. Crit. Care Med. 2009, 37, 177-183. [CrossRef] 